\title{
Coherent UD-WDM RoF Fronthaul Network with D-EML Transmitter and Phase-Noise Robust Receiver
}

\author{
Miquel Masanas, Jeison Tabares, Josep Prat, Member, IEEE
}

\begin{abstract}
This work proposes and tests a simplified coherent uplink architecture for ultra-dense coherent analog mobile Fronthaul with phase noise cancellation based on single sideband modulation of a dual-EML. Results show optical channel spacings of only $1.5 \mathrm{GHz}$ and error-free signal recovery with $\Delta v \mathrm{~Tb}=1.2 \%$ and $3 \%$ with reaching power as low as $-40 \mathrm{dBm}$ for $100 \mathrm{Mbit} / \mathrm{s}$ and -37 dBm for $1 \mathrm{Gbit} / \mathrm{s}$ using QPSK modulation.
\end{abstract}

Index Terms - Coherent radio-over-fiber (CRoF), coherent mobile Fronthaul, dual-electroabsorption-modulated laser (DEML), optical communications, optical sources, optical single sideband (OSSB), phase-noise cancelling, fiber-wireless, mobile Fronthaul, radio-over-fiber.

\section{INTRODUCTION}

$\mathrm{D}$ ISTRIBUTED antenna systems (DAS) for low range operation have been proposed both for $5 \mathrm{G}$ Fronthaul and to solve increasing congestion in Wi-Fi bands while operating at the same frequencies [1]. Early deployments of 5G have used low-cost gray optics, but this solution is not scalable to dense urban areas using a DAS approach, which demands higher Fronthaul connectivity. Also, future systems need to deliver high performance, linearity, integration and low phase noise at once [2,3]. Proposals of ultra-dense wavelength division multiplexing (UD-WDM) radio access networks (RAN) using wavelength splitting have been proposed in [4], [5]. However, it is likely that areas where new deployments are unfeasible will need to use existing passive optical networks (PONs) and dark fibres. The need to use legacy networks requires Fronthaul connectivity through colourless networks and the use of high splitting ratios. This places stringent requirements on the power budgets and spectrum management to achieve.

We propose reaching the Fronthaul connectivity demands through coherent UD-WDM. Coherent detection improves greatly the receiver sensitivity, as well as user bandwidth (BW) allocation through wavelength multiplexing [6], but leads to high phase noise. We implement phase noise cancelling (PNC) by optical heterodyne detection of an optical single sideband full-carrier (OSSB-FC) modulation followed by an electrical

Manuscript received July XX, 2021; accepted XX. This work was supported in part by the Spanish CICYT project VERSONET under id. RTI2018-097051 and by grant "Proyectos de I+D+i" with id. PID2019-107885GB-C31. (Corresponding author: Miquel Masanas.)

The authors are with the Department of Signal Theory and Communications, Universitat Politècnica de Catalunya, Barcelona 08034, Spain heterodyne detection between the data and the carrier, which contains phase information of both transmitter (Tx) and receiver $(\mathrm{Rx})$ laser sources $[7,8]$. The OSSB-FC is achieved by using a dual-electroabsorption-modulated laser (D-EML), resulting in high-quality $\mathrm{RF}$ generation and detection using low-cost hardware and maintaining maximum optoelectronic integration. We test for the first time, to the best of our knowledge, this simple combination of D-EML transmitter and coherent receiver solution with PNC for mobile Fronthaul. We do it with low data rates and high linewidth $(\Delta v)$ optical sources.

The experiments are done using QPSK at data rates of $1 \mathrm{~Gb} / \mathrm{s}$, used in $5 \mathrm{G}$, and only $100 \mathrm{Mbit} / \mathrm{s}$, as used in $4 \mathrm{G}$, over a $1 \mathrm{GHz}$ electrical carrier, testing remarkably disadvantageous conditions in terms of phase noise for coherent systems. The proposal is directly scalable to higher carrier frequencies, data rates and modulation orders, as being considered by $5 \mathrm{G}$ operators.

\section{LINK PROPOSAL}

The proposal for the uplink transmission in the RAN Fronthaul is depicted in Fig. 1. The antenna Tx consists of a DEML in OSSB-FC configuration (Fig. 2a). To obtain this modulation, the distributed feedback (DFB) laser section of the D-EML is modulated as a FM modulator, while the electroabsorption section provides IM modulation. This concatenation results in asymmetric harmonic cancellation for certain modulation indexes. For small modulation indexes, the OSSB-FC of a single tone is obtained in the first harmonic when the IM modulation index is twice the FM modulation index [9], [10]. At the Rx a single coherent heterodyne detection front-end is used to detect several UD-WDM channels, decreasing the usual complexity of coherent detections with many local oscillators (LOs).

At the electrical level, signal and carriers are separated with RF band-pass filters ( Fig. 2b), and each carrier is re-beaten with its corresponding signal in a second electrical heterodyne demodulation, cancelling the phase noise and frequency drifts.

(e-mail:miquel.masanas@upc.edu; jtabares@tsc.upc.edu; jprat@tsc.upc.edu). Copyright (c) 2019 IEEE. Personal use of this material is permitted. However, permission to use this material for any other purposes must be obtained from the IEEE by sending a request to pubs-permissions@ieee.org. 


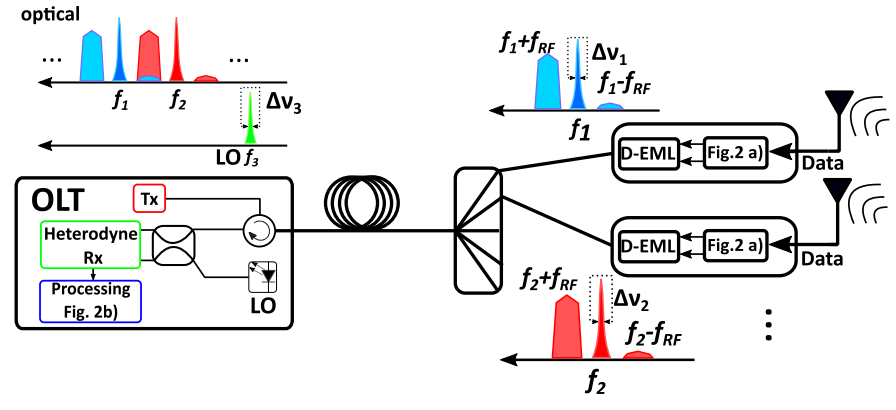

Fig. 1: Proposed Fronthaul link over PON.

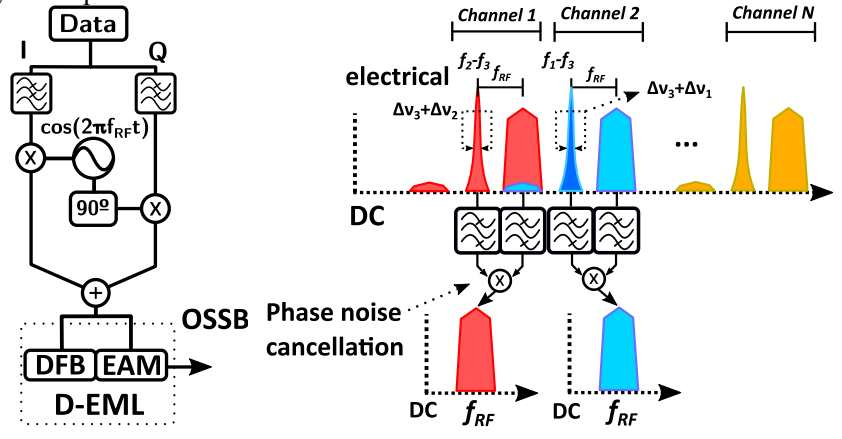

a)

b)

Fig. 2: a) Modulation for OSSB-FC. b) Electrical second heterodyne detection for phase noise and detuning cancelling.

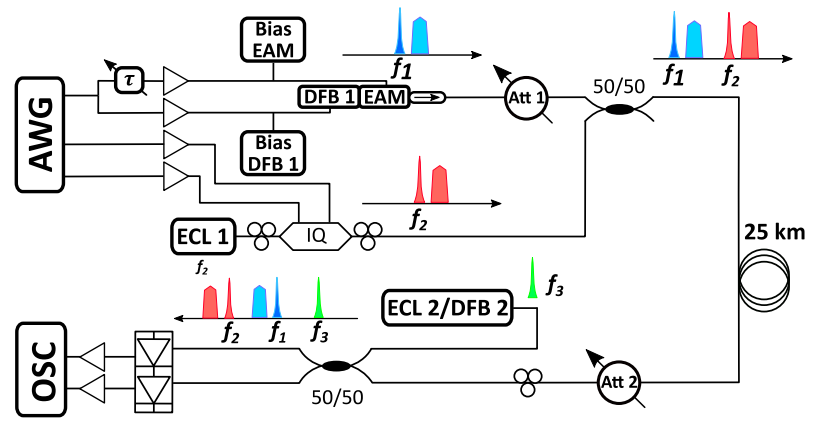

a)

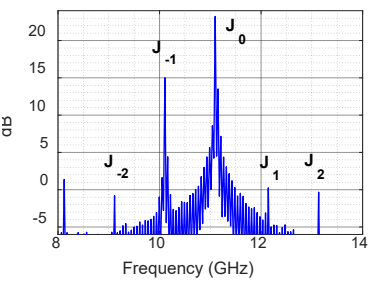

b)

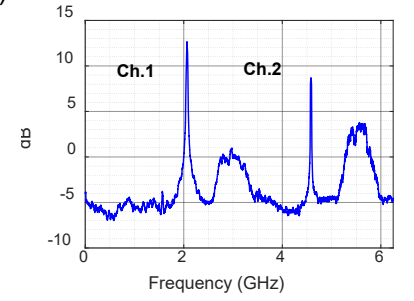

c)
Fig. 3: a) Experimental set-up. AWG: Arbitrary wave generator. OSC: oscilloscope. b) SSB-FC with RF tone modulation. c) Detected multichannel spectra.

This results in a phase-noise clean data channel over the original modulating frequency $\mathrm{f}_{\mathrm{RF}}$. In order to increase spectral efficiency, in signals using tenths of $\mathrm{GHz}$ for their carriers, the spectral distribution scheme in Fig. 2b) could use channel interleaving [11] rather than a sequential ordering of them, as the carriers and their signals do not need to be consecutive for the technique to work.
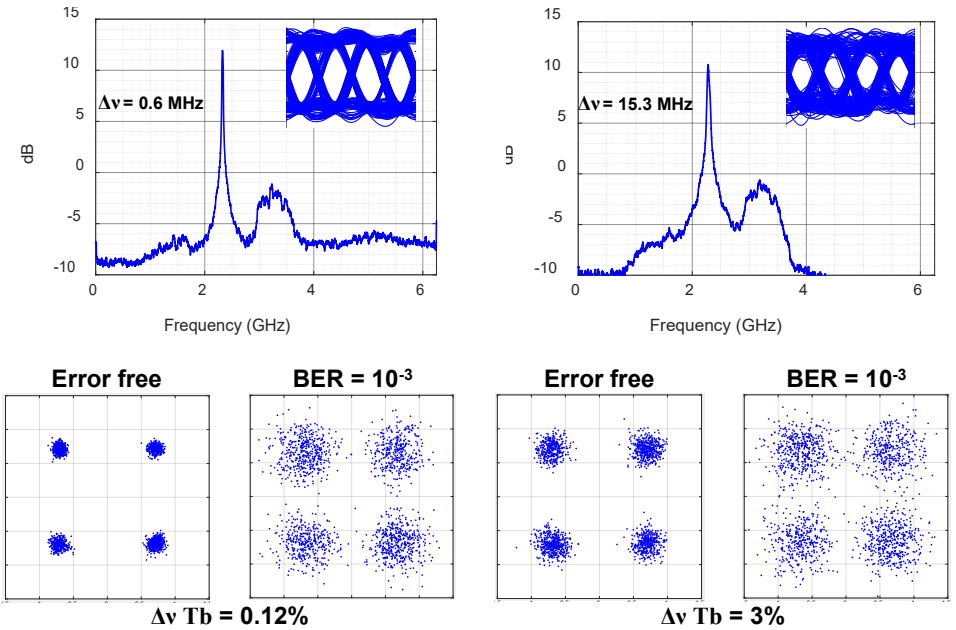

Fig. 4: Spectrums, eye and constellation diagrams of the single channel scenario using ECL 2 (left) and DFB 2 (right) as LO for $\mathrm{Rb}=500 \mathrm{Mbaud}$, resulting in $\Delta v \mathrm{~Tb}=$ $0.12 \%, 3 \%$ respectively, with $\mathrm{Tb}$ the symbol period. Eye diagrams at the same received power.
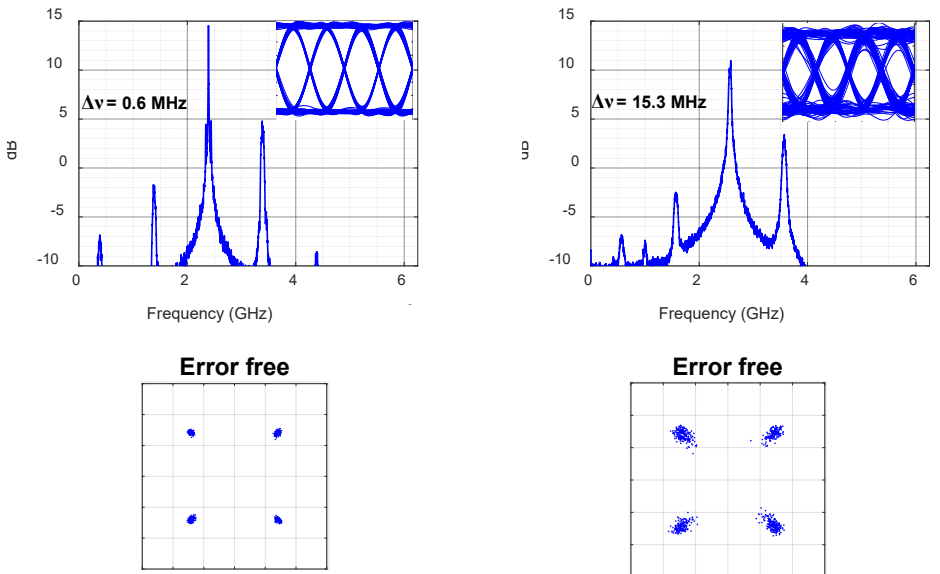

$\Delta v \mathrm{~Tb}=1.2 \%$

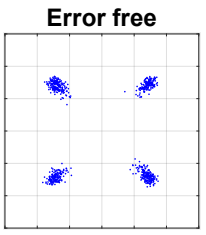

$\Delta \mathbf{v} \mathbf{T b}=\mathbf{3 0} \%$

Fig. 5: Idem Fig. 4 for $\mathrm{Rb}=50$ Mbaud, resulting in $\Delta v \mathrm{~Tb}=1.2 \%, 30 \%$ respectively, with $\mathrm{Tb}$ the symbol period.

\section{EXPERIMENTAL SETUP}

To test the proposal, the setup in Fig. 3a) was built. It consists in two transmitters: channel 1 (ch.1) being the main one to be evaluated, implementing a D-EML using distributed feedback (DFB) laser 1 of $\Delta v=300 \mathrm{kHz}$, and channel 2 (ch.2) based on an optical IQ modulator based on a Dual-Parallel MachZehnder Modulator (DP-MZM) and external cavity laser (ECL 1) with $\Delta v=100 \mathrm{kHz}$. The OSSB-FC, seen in Fig. 3b) for 1 $\mathrm{GHz}$ RF tone modulation, is obtained by placing the electroabsorption modulator (EAM) section at the maximum linearity region and modulating the EAM and DFB sections with $\mathrm{m}_{\mathrm{IM}}=$ 0.12 and $\mathrm{m}_{\mathrm{FM}}=0.05$, with extinction ratio between $\mathrm{J}_{-1} / \mathrm{J}_{1}$ of 15 $\mathrm{dB}$, and between $\mathrm{J}_{0} / \mathrm{J}_{-1}$ of $8 \mathrm{~dB}$, where $\mathrm{J}_{\mathrm{n}}$ represents the Bessel function of order $n$. Higher order harmonics are further reduced. Increasing modulation depths results in the rise of said higher order harmonics and a non-linear performance of the EAM, leading to a shift of the optimum SSB operation point.

The oscilloscope used for in-line demodulation is a Tektronix DPO71254B operated at $12.5 \mathrm{GSa} / \mathrm{s}$ with an electrical bandwidth of $6 \mathrm{GHz}$. 


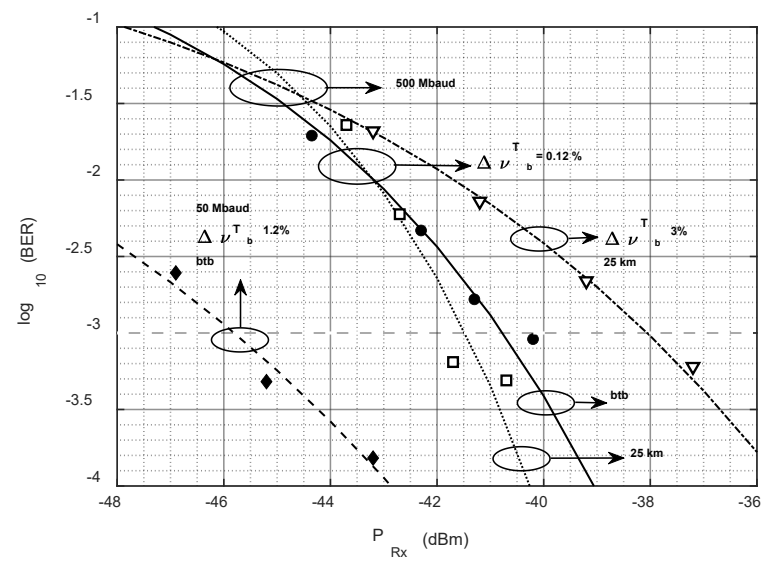

Fig. 6: Measured BER against reaching power for $\mathrm{Rb}=50,500 \mathrm{Mbaud}$ and $\Delta v=0.6 \mathrm{MHz}, 15.3 \mathrm{MHz}$.

The photodetection was done using a pair of $10 \mathrm{GHz}$ PIN+TIA with a responsivity of $20 \mathrm{~A} / \mathrm{W}$, including the TIA gain.

Fig 3c) shows the balanced photodetected signal at the receiver for the multichannel case with symbol rates $(\mathrm{Rb})$ of 500 Mbaud using an ECL 2 with $\Delta v=300 \mathrm{kHz}$ as a LO. They are optically separated $2.5 \mathrm{GHz}$. The modulation of ch.1 has been obtained for $\mathrm{m}_{\mathrm{IM}}=0.4$ and $\mathrm{m}_{\mathrm{FM}}=0.1$, again in the maximum linearity region of the EAM, and the modulation of ch.2 has been generated by operating the IQ modulator in OSSB configuration $[12,13]$, with modulation biases shifted from the null point so a residual carrier is left, obtaining a carrier-tosignal ratio of about $5 \mathrm{~dB}$ to approximate the modulation of the D-EML. The signals of ch.1 and ch.2 were uncorrelated in time by different physical path differences. The tests have been done using $\mathrm{Rb}$ of 50 and 500 Mbaud at a QPSK configuration with a modulating carrier of frequency $\mathrm{f}_{\mathrm{RF}}=1 \mathrm{GHz}$ and square root raised cosine filtering at the Tx and Rx with roll-off factor of 1 . The signals are combined using a $3 \mathrm{~dB}$ optical coupler and travel $25 \mathrm{~km}$ of standard single-mode fibre. Att. 1 ensures that channels have equal power. The launch power of ch.1 for the single channel BER measurements is $0 \mathrm{dBm}$.

The resulting signal reaches a standard heterodyne $2 \times 2$ optical front end with LO power of $0.5 \mathrm{dBm}$. Then, in-line processing of the signals is used to obtain the balanced signal. Both data and carrier are recovered using square frequency band pass filters of BW $200 \mathrm{MHz}$ for the carrier and either 50 or $500 \mathrm{MHz}$ for the data recovery. Then, electrical heterodyne detection is performed using said components as in Fig. 2b), resulting in a clean signal at $f_{R F}$. Finally, down conversion to base-band for bit error ratio (BER) evaluation is done. To test the resilience against phase noise, DFB 2 with $\Delta v=15 \mathrm{MHz}$ has been also used as a $\mathrm{LO}$ for testing, at a power of $0.3 \mathrm{dBm}$. In the presented experiments, state of polarization (SOP) is controlled manually. In real deployments, either a conventional polarization diversity heterodyne front-end or even our more efficient novel alternative with image frequency rejection and reduced hardware can readily be used [14].

\section{RESULTS}

Fig. 4 and 5 show the single channel photodetected signal spectra before electrical heterodyning for both 500 and 50

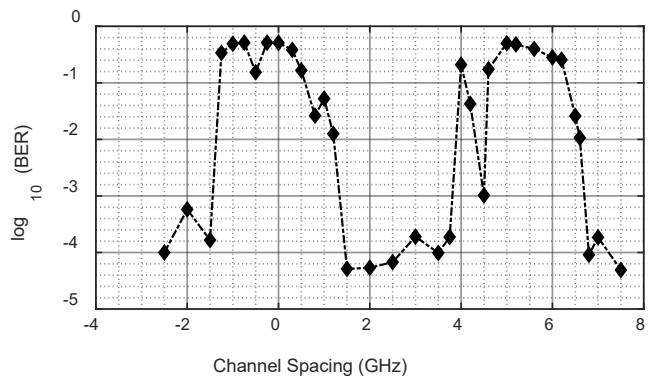

a)

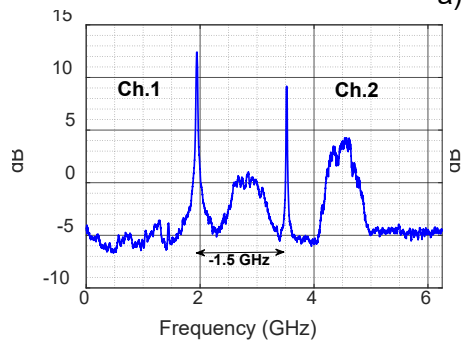

b)

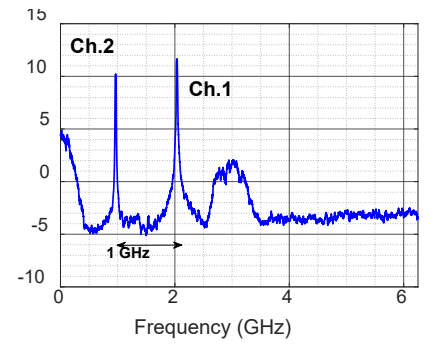

c)
Fig. 7: a) BER penalty with channel spacing. b,c) Received spectra with channel spacing $-1.5,1 \mathrm{GHz}$.

Mbaud using as LO both ECL 2 and DFB 2, and the eye and constellation diagrams for said configurations. From the eye diagrams and error-free constellation diagrams it can be seen how a broader $\Delta v$ results in symbol distortion. This can be explained by the fact that the band-pass filters limit the recovery of some phase noise for high linewidth, leading to residual distortion over the target signal. The distortion does not result in the circular phase rotation of the symbols, but rather in a radial scattering. Thus, BER penalty can be expected with increasing $\Delta v$, but the constellation can be recovered error-free even for a $\Delta v \mathrm{~Tb}$ as high as $30 \%$, with $\mathrm{Tb}$ being the symbol period, as seen in Fig. 5 right.

Fig. 6 presents the BER performance of the single channel studied cases for different linewidth LOs and bit rates. For a reference BER of $10^{-3}$, sensitivity for the 50 Mbaud in back to back (btb) configuration is $-46 \mathrm{dBm}$ with a $\Delta v \mathrm{~Tb}$ of $1.2 \%$. Sensitivity for the 500 Mbaud scenario with ECL 2 as LO ( $\Delta v$ $\mathrm{Tb}$ of $0.12 \%$ ) result in $-40.8 \mathrm{dBm}$ in btb and $-41.5 \mathrm{dBm}$ in 25 $\mathrm{km}$ fiber span respectively. Finally, the sensitivity for 500 Mbaud, $25 \mathrm{~km}$ fibre span and DFB 2 as LO ( $\Delta v$ Tb of $3 \%$ ) results in $-38 \mathrm{dBm}$, which compared with the previous $\mathrm{Rb}=500$ Mbaud cases has a 2.8 penalty with respect the btb measure and $3.5 \mathrm{~dB}$ penalty with respect the $25 \mathrm{~km}$ span measure. The bitenergy difference between the 50 and 500 Mbaud curves resulted in $5.2 \mathrm{~dB}$ difference with the btb curve and $4.5 \mathrm{~dB}$ penalty with the $25 \mathrm{~km}$ span curve. Other QPSK systems based on carrier recovery through phase estimation have $\Delta v \mathrm{~Tb}<$ $0.041 \%$ [15].

Next, another user (ch.2) is added for channel spacing evaluation. Fig. 7a) shows the BER penalty over channel spacing between ch.1 and ch.2 with ECL 2 as LO with intermediate frequency (fIF) $2 \mathrm{GHz}$. Then, channel 2 is swept from frequencies $5.5 \mathrm{GHz}$ below the $\mathrm{LO}$, or $7.5 \mathrm{GHz}$ below 
ch.1, to $4.5 \mathrm{GHz}$ above the $\mathrm{LO}$, or $2.5 \mathrm{GHz}$ above ch.1. We use $500 \mathrm{Mbaud} \mathrm{Rb}$, as well as $25 \mathrm{~km}$ of fibre. Channel spacing is defined as the difference between optical frequencies of ch.1 and ch. 2 carriers. Total optical overlap of both channel carriers $\mathrm{J}_{0}$ and data bands happens at $0 \mathrm{GHz}$. At $5 \mathrm{GHz}$, ch.2 carrier overlaps ch.1 data, and its data band overlaps ch.1 carrier due to image frequency interference. For the same reason, total overlap between channel carriers also happens at $4 \mathrm{GHz}$ and total overlap between ch.1 carrier and ch.2 data band happens at $1 \mathrm{GHz}$. Optical overlap between ch. 1 data band and ch. 2 carrier happens at $-1 \mathrm{GHz}$. These interferences result in signal destruction. At $4.5 \mathrm{GHz}$ the image frequency interference from the carrier of ch. 2 lies between the data band and carrier of ch.1, and its data band interferes only with ch.1 carrier, leading to a lower interference. In contrast, around channel spacing -0.5 $\mathrm{GHz}$ only a slight BER improvement is observed as ch.2 data band interferes strongly with ch.1 data band.

The minimum channel spacing between channels received by the same Rx is $-1.5 \mathrm{GHz}$ (Fig. 7b), which matches the data band $\mathrm{BW}$ plus the modulating $\mathrm{f}_{\mathrm{RF}}$. A channel spacing of $-2 \mathrm{GHz}$ between channels of the same receiver ensures good performance with frequency drift, although there is the penalty from the non-ideal cancellation of the $\mathrm{J}_{-1}$ sideband. Due to image frequency interference, the minimum channel spacing between channel groups detected by two adjacent Rx is the sum of both Rx BWs. Considering now ch.2 as an interfering signal from the previous $\mathrm{Rx}$, and the evaluated $\mathrm{Rx} \mathrm{BW}=3.5 \mathrm{GHz}$, corresponding to the $\mathrm{f}_{\mathrm{IF}}$ plus ch.1 BW, Fig. 7a) correctly predicts a channel spacing between $\mathrm{Rx}$ of $7 \mathrm{GHz}$ to avoid image frequency interference.

Interestingly, no penalty is observed when ch.2 interferes with the LO (Fig. 7c). Total overlap between ch.2 carrier and LO happens at $2 \mathrm{GHz}$, and total overlap between $\mathrm{LO}$ and ch. 2 data band happens at $3 \mathrm{GHz}$, resulting in an image frequency of ch.2 carrier at $1 \mathrm{GHz}$ as expected. No penalty is observed because when channel 2 overlaps the LO, the interference affects both the recovery of channel 1 carrier and its data band, and through the second heterodyne mixing shown in Fig. 2b), the interference is compensated.

The used D-EML chip in this study is unpackaged and manually aligned. Misalignment over time and limited repeatability results in observed BER fluctuations of $<1 \mathrm{~dB}$ between the $25 \mathrm{~km}$ span and btb curves for the 500 Mbaud curves.

\section{CONCLUSIONS}

In this work, significant sensitivities of $-38 \mathrm{dBm}$ for 500 Mbaud with $\Delta v \mathrm{~Tb}=3 \%$ and $-46 \mathrm{dBm}$ for 50 Mbaud with $\Delta v$ $\mathrm{Tb}=1.2 \%$ are reported using a simple combination of a DEML in OSSB-FC configuration and heterodyne receiver. Channel spacing between channels detected in the same $\mathrm{Rx}$ is as low as $\mathrm{f}_{\mathrm{RF}}+\mathrm{Rb}=1.5 \mathrm{GHz}$ with root raised cosine pulse shaping of roll-off factor 1, enabling UD-WDM schemes over splitter-based PONs.

In short, the work demonstrates efficient resource use for next generation ud-WDM coherent RoF with high integration, phase noise cancelling and both capacity and power budget enhancement.

\section{ACKNOWLEDGEMENTS}

The authors would like to thank the Spanish CICYT projects VERSONET (RTI2018-097051) and Catalan GRC-UPC-GCO L-0625.

\section{REFERENCES}

[1] Paredes-Páliz, et al., 2020. "Radio over Fibre: An Alternative Broadband Network Technology for IoT" Electronics 9, no. 11: 1785. https://doi.org/10.3390/electronics9111785

[2] FG IMT-2020: Report on Standards Gap Analysis, International Telecommunication Union (ITU)-Telecommunication Standardization Sector, Geneva, 2016.

[3] C. Lim, et al., "Evolution of Radio-Over-Fibre Technology," in Journal of Lightwave Technology, vol. 37, no. 6, pp. 1647-1656, 15 March15, 2019, doi: 10.1109/JLT.2018.2876722.

[4] Jim (Shihuan) Zou et al., "Advanced optical access technologies for next-generation (5G) mobile networks [Invited]," J. Opt. Commun. Netw. 12, D86-D98, 2020.

[5] D. Konstantinou, et al, 5G RAN architecture based on analog radioover-fibre fronthaul over UDWDM-PON and phased array fed reflector antennas, Optics Communications, Volume 454, 2020, 124464, ISSN 0030-4018, https://doi.org/10.1016/j.optcom.2019.124464.

[6] J. Prat et al., "Technologies for Cost-Effective udWDM-PONs," in Journal of Lightwave Technology, vol. 34, no. 2, pp. 783-791, 15 Jan.15, 2016, doi: 10.1109/JLT.2015.2499381

[7] M. Presi, M. Rannello and E. Ciaramella, "A homodyne coherent receiver for analog-over-fibre systems based on feed-forward phasenoise cancellation," 45th European Conference on Optical Communication, ECOC 2019, 2019, pp. 1-4, doi: 10.1049/cp.2019.0778.

[8] C. Browning, et al., "Phase noise robust optical heterodyne system for reduced complexity millimeter-wave analog radio-over-fibre," 45 th European Conference on Optical Communication (ECOC 2019), 2019, pp. 1-4, doi: 10.1049/cp.2019.0779.

[9] H. Kim, "EML-Based Optical Single Sideband Transmitter," in IEEE Photonics Technology Letters, vol. 20, no. 4, pp. 243-245, Feb.15, 2008, doi: 10.1109/LPT.2007.913333.

[10] D. Erasme et al., "The Dual-Electroabsorption Modulated Laser, a Flexible Solution for Amplified and Dispersion Uncompensated Networks Over Standard Fibre," in Journal of Lightwave Technology, vol. 32, no. 21, pp. 4068-4078, 1 Nov.1, 2014, doi: 10.1109/JLT.2014.2346427.

[11] Konstantinou, Dimitrios \& Bressner, Thomas \& Rommel, Simon \& Johannsen, Ulf \& Johansson, Martin \& Ivashina, M. \& Smolders, A. \& Tafur Monroy, Idelfonso. (2019). 5G RAN architecture based on analog radio-over-fiber fronthaul over UDWDM-PON and phased array fed reflector antennas. Optics Communications. 454. 124464. 10.1016/j.optcom.2019.124464.

[12] X. Chen and J. Yao, "A High Spectral Efficiency Coherent RoF System Based on OSSB Modulation With Low-Cost Free-Running Laser Sources for UDWDM-PONs," in Journal of Lightwave Technology, vol. 34, no. 11, pp. 2789-2795, 1 June1, 2016.

[13] M. Masanas, et al., "Rayleigh Backscattering Rejection in SingleLaser Homodyne Transceiver for UD-WDM Using $\lambda$-Shifting," in IEEE Photonics Technology Letters, vol. 33, no. 7, pp. 354-357, 1 April1, 2021, doi: 10.1109/LPT.2021.3061774.

[14] J. Tabares and J. Prat, "Low-Complexity Phase-and-PolarizationDiversity Coherent Receiver with High Spectral Efficiency for UDWDM," in Proc. Opt. Fiber Commun. Conf. Exhib., 2021 (OFC), Paper Th5I.2.

[15] T. Pfau, S. Hoffmann, and R. Noé, "Hardware-Efficient Coherent Digital Receiver Concept With Feedforward Carrier Recovery for MQAM Constellations," Journal of Lightwave Technology, vol. 27, no. 8, pp. 989, 2009. 